\title{
The Voigt Profile as a Sum of a Gaussian and a Lorentzian Functions, when the Weight Coefficient Depends on the Widths Ratio and the Independent Variable
}

\author{
H.O. Di RocCO ${ }^{a, b, *}$ AND A. CRuzado ${ }^{c, d}$ \\ ${ }^{a}$ Instituto de Física Arroyo Seco (IFAS), Universidad Nacional del Centro de la Pcia. de Buenos Aires \\ Pinto 399, 7000 Tandil, Buenos Aires, Argentina \\ ${ }^{b}$ Consejo Nacional de Investigaciones Científicas y Técnicas (CONICET) \\ Rivadavia 1917, C1033AAJ Buenos Aires, Argentina \\ ${ }^{c}$ Facultad de Ciencias Astronómicas y Geofísicas, Universidad Nacional de La Plata, Paseo del Bosque s/n \\ 1900 La Plata, Buenos Aires, Argentina \\ ${ }^{d}$ Instituto de Astrofísica de La Plata (IALP-CONICET), Paseo del Bosque s/n \\ 1900 La Plata, Buenos Aires, Argentina
}

(Received December 20, 2011; revised version March 6, 2012; in final form May 24, 2012)

\begin{abstract}
Assuming that $V(x) \approx(1-\mu) G_{1}(x)+\mu L_{1}(x)$ is a very good approximation of the Voigt function, in this work we analytically find $\mu$ from mathematical properties of $V(x) . G_{1}(x)$ and $L_{1}(x)$ represent a Gaussian and a Lorentzian function, respectively, with the same height and HWHM as $V(x)$, the Voigt function, $x$ being the distance from the function center. In this paper we extend the analysis that we have done in a previous paper, where $\mu$ is only a function of $a$; $a$ being the ratio of the Lorentz width to the Gaussian width. Using one of the differential equation that $V(x)$ satisfies, in the present paper we obtain $\mu$ as a function, not only of a, but also of $x$. Kielkopf first proposed $\mu(a, x)$ based on numerical arguments. We find that the Voigt function calculated with the expression $\mu(a, x)$ we have obtained in this paper, deviates from the exact value less than $\mu(a)$ does, specially for high $|x|$ values.
\end{abstract}

PACS: $32.70 .-\mathrm{n}, 32.70 . \mathrm{Jz}$

\section{Introduction}

Let $V_{a}(x)$ be the Voigt profile obtained by convolution of $G(x)$ with $L(x)$.

Approximating the Voigt profile as

$$
V_{a}(x) \approx(1-\mu) G_{1}(x)+\mu L_{1}(x),
$$

in a previous paper [1] (PI hereafter), we analytically found $\mu$ as a function only on $a$, using the property of the normalized area.

In PI we did some general considerations showing that, using the expression (1) to represent the Voigt profile, we would rigorously calculate $\mu$ for each $x$ value, to obtain $\mu$ as a function, not only on $a$, but also on $x$, as follows:

$$
\mu(a, x)=\frac{V_{a}(x)-G_{1}(x)}{L_{1}(x)-G_{1}(x)} .
$$

Even though the deviations of $V_{a}(x)$ calculated with $\mu(a)$ from its exact values are smaller than $0.5 \%$ relative to the peak value, as it was found in PI, it is useful, theoretically and practically, to calculate $\mu(a, x)$ from Eq. (2).

It is important to emphasize that, although Eq. (2) can be calculated for each $x$ value for every profile $V_{a}(x)$, there is not a simple analytical expression for the right

\footnotetext{
* corresponding author; e-mail: hdirocco@exa.unicen.edu.ar
}

hand side of this expression. Indeed, if we expand in series, we see that the coefficients of $V_{a}(x)$ depend complicatedly on $a$, involving Kummer functions (or confluent hypergeometric functions), as was shown in [2].

The dependence on $x$ was already introduced by Kielkopf [3] based on numerical arguments. In the present paper we find an analytical expression for $\mu(a, x)$ from one of the differential equation that $V(x)$ satisfies, which allow a better fit of $V_{a}(x)$ than $\mu(a)$ does, specially for high $|x|$ values.

We emphasize that, beyond the usefulness, this semitheoretical approach produces, in our opinion, a certain aesthetic satisfaction.

\section{Analytical deduction of $\mu(a, x)$ using one of the differential equations satisfied by $V_{a}(x)$}

$V_{a}(x)$ satisfies several differential equations, that can be found in [4] and [5]. Of direct usefulness in our case is the following:

$$
V^{\prime \prime}(x)+\frac{4 x V^{\prime}(x)}{w_{\mathrm{G}}^{2}}+\left(\frac{4 a^{2}+2}{w_{\mathrm{G}}^{2}}+\frac{4 x^{2}}{w_{\mathrm{G}}^{4}}\right) V(x)=\frac{4 a}{\pi w_{\mathrm{G}}^{3}} .
$$

If $\mu=\mu(a, x)$ is assumed in Eq. (1), Eq. (3) results very complicated to our purposes, with terms like $\partial \mu / \partial x$ and $\partial^{2} \mu / \partial x^{2}$, and other such as $G_{1}^{\prime}(x), G_{1}^{\prime \prime}(x)$, 
$L_{1}^{\prime}(x)$ and $L_{1}^{\prime \prime}(x)$. But, if the derivatives like $\partial \mu / \partial x$ and $\partial^{2} \mu / \partial x^{2}$ are negligible with respect to the derivatives $G_{1}^{\prime}(x), G_{1}^{\prime \prime}(x), L_{1}^{\prime}(x)$ and $L_{1}^{\prime \prime}(x)$, Eq. (3) becomes simple enough to obtain a function $\mu(a, x)$ that permits a better fit as compared with which has been obtained in PI, specially for high $|x|$ values.

Taking into account that the derivatives $G_{1}^{\prime}(x)$ and $G_{1}^{\prime \prime}(x)$ are proportional to $G_{1}(x)$ and that $L_{1}^{\prime}(x)$ and $L_{1}^{\prime \prime}(x)$ are proportional to $L_{1}(x)$, from Eq. (3) we obtain

$$
\begin{aligned}
- & \frac{2 \ln 2(1-\mu) G_{1}(x)}{\gamma_{\mathrm{V}}^{2}}+\frac{4(\ln 2)^{2}(1-\mu) x^{2} G_{1}(x)}{\gamma_{\mathrm{V}}^{4}} \\
& +\frac{8 \mu x^{2} L_{1}(x)}{\left(x^{2}+\gamma_{\mathrm{V}}^{2}\right)^{2}}-\frac{2 \mu L_{1}(x)}{x^{2}+\gamma_{\mathrm{V}}^{2}} \\
& +4 x\left[-\frac{2 \ln 2(1-\mu) x G_{1}(x)}{w_{\mathrm{G}}^{2} \gamma_{\mathrm{V}}^{2}}-\frac{2 \mu x L_{1}(x)}{w_{\mathrm{G}}^{2}\left(x^{2}+\gamma_{\mathrm{V}}^{2}\right)}\right] \\
& +P(x)\left[(1-\mu) G_{1}(x)+\mu L_{1}(x)\right]=Q
\end{aligned}
$$

with $P(x)=\left(4 a^{2}+2\right) / w_{\mathrm{G}}^{2}+4 x^{2} / w_{\mathrm{G}}^{4}, Q=4 a /\left(\pi w_{\mathrm{G}}^{3}\right)$ and $\mu=\mu(a, x)$. Developing the former expression and collecting terms, we obtain

$$
\mu(a, x)=\frac{f_{\mathrm{G}}(x) G_{1}(x)+f_{\mathrm{Q}}(x) Q}{f_{\mathrm{L}}(x) L_{1}(x)+f_{\mathrm{G}}(x) G_{1}(x)},
$$

where

$$
\begin{aligned}
& f_{\mathrm{Q}}(x)=\gamma_{\mathrm{V}}^{8} w_{\mathrm{G}}^{2}+2 \gamma_{\mathrm{V}}^{6} w_{\mathrm{G}}^{2} x^{2}+\gamma_{\mathrm{V}}^{4} w_{\mathrm{G}}^{2} x^{4} \\
& f_{\mathrm{G}}(x)=-8(\ln 2)^{2} \gamma_{\mathrm{V}}^{2} w_{\mathrm{G}}^{2} x^{4}-4(\ln 2)^{2} \gamma_{\mathrm{V}}^{4} w_{\mathrm{G}}^{2} x^{2} \\
& \quad+2(\ln 2) \gamma_{\mathrm{V}}^{6} w_{\mathrm{G}}^{2}-2 P(x) \gamma_{\mathrm{V}}^{6} w_{\mathrm{G}}^{2} x^{2}-P(x) \gamma_{\mathrm{V}}^{4} w_{\mathrm{G}}^{2} x^{4} \\
& \quad-4(\ln 2)^{2} w_{\mathrm{G}}^{2} x^{6}+8(\ln 2) \gamma_{\mathrm{V}}^{6} x^{2}+16(\ln 2) \gamma_{\mathrm{V}}^{4} x^{4} \\
& \quad+8(\ln 2) \gamma_{\mathrm{V}}^{2} x^{6}-P(x) \gamma_{\mathrm{V}}^{8} w_{\mathrm{G}}^{2}+4(\ln 2) \gamma_{\mathrm{V}}^{4} w_{\mathrm{G}}^{2} x^{2} \\
& \quad+2(\ln 2) \gamma_{\mathrm{V}}^{2} w_{\mathrm{G}}^{2} x^{4}
\end{aligned}
$$

and

$$
\begin{aligned}
& f_{\mathrm{L}}(x)=6 \gamma_{\mathrm{V}}^{4} w_{\mathrm{G}}^{2} x^{2}-2 \gamma_{\mathrm{V}}^{6} w_{\mathrm{G}}^{2}+2 P(x) \gamma_{\mathrm{V}}^{6} w_{\mathrm{G}}^{2} x^{6} \\
& \quad+P(x) \gamma_{\mathrm{V}}^{4} w_{\mathrm{G}}^{2} x^{4}+P(x) \gamma_{\mathrm{V}}^{8} w_{\mathrm{G}}^{2}-8 \gamma_{\mathrm{V}}^{6} x^{2}-8 \gamma_{\mathrm{V}}^{4} x^{4} .
\end{aligned}
$$

In spite of neglecting the derivatives $\partial \mu / \partial x$ and $\partial^{2} \mu / \partial x^{2}$, the $\mu(a, x)$ values we obtain from Eq. (5) depart very little from those we obtain from Eq. (2).

\subsection{Limit cases}

In order to achieve the final analytic expression we are searching, we need to know the behavior of $\mu(a, x)$ near $x=0$ and for $x \rightarrow \infty$.

Taking into account that $\mu(a, x)$ is an even function of $x$, we analyse those limit cases in the following sections.

2.1.1. $\mu(a, x)$ value at $x=0$

Evaluating $f_{\mathrm{G}}(x), f_{\mathrm{L}}(x)$ and $f_{\mathrm{Q}}(x)$ at $x=0$, Eq. (5) can be written as

$$
\begin{gathered}
\mu(a, 0)=-2.258891354+\frac{6.517782707 a^{2} \gamma_{\mathrm{V}}^{2}}{w_{\mathrm{G}}^{2}} \\
+\frac{3.258891354 \gamma_{\mathrm{V}}^{2}}{w_{\mathrm{G}}^{2}}-\frac{2.888117265 \times 4 a \times \gamma_{\mathrm{V}}^{3}}{\pi w_{\mathrm{G}}^{3}} .
\end{gathered}
$$

Taking into account that $\gamma_{\mathrm{V}} / w_{\mathrm{G}}=b_{1 / 2}(a)$, as we see in PI, we finally obtain

$$
\begin{aligned}
& \mu(a, 0)=-2.258891354+6.517782707 a^{2} b_{1 / 2}^{2}(a) \\
& +3.258891354 b_{1 / 2}^{2}(a) \\
& -\frac{2.888117265 \times 4 a \times b_{1 / 2}^{3}(a)}{\pi} .
\end{aligned}
$$

Comparing $\mu(a, 0)$ given by the former expression with $\mu(a)$ obtained in PI and given by

$$
\mu(a)=\frac{b_{1 / 2}(a) \mathrm{e}^{a^{2}} \Phi_{\mathrm{c}}(a)-\sqrt{\ln (2)}}{b_{1 / 2}(a) \mathrm{e}^{a^{2}} \Phi_{\mathrm{c}}(a)(1-\sqrt{\pi \ln (2)})},
$$

we conclude that $\mu(a, 0)<\mu(a)$ for every $a$ value.

\subsection{2. $\mu(a, x)$ value near $x=0$}

It is known that a even function can be expanded as an even power series about zero as $f(x) \simeq A_{0}+A_{1} x^{2}+\ldots$ But, since $\mu(a, x)$ is expressed as a quotient (Eq. (5)), we expand numerator and denominator to obtain $\mu(a, x)$ as a quotient of power series about zero.

Then, at $x \simeq 0$ it is verified

$$
\mu(a, x \rightarrow 0)=\frac{\mu_{\mathrm{app}}(a, 0)+A x^{2}\left(+B x^{4}+\ldots\right)}{1+C x^{2}\left(+D x^{4}+\ldots\right)} .
$$

\subsection{3. $\mu(a, x)$ value at $x \rightarrow \infty$}

Taking into account that $G_{1}(x)$ tends to zero much faster than $Q$ and $L_{1}(x)$, from Eq. (5) it is obtained

$$
\lim _{x \rightarrow \infty} \mu(a, x) \sim \lim _{x \rightarrow \infty} \frac{f_{\mathrm{Q}}(x) Q}{f_{\mathrm{L}}(x) L_{1}(x)},
$$

where

$$
\begin{aligned}
& \lim _{x \rightarrow \infty} f_{\mathrm{Q}}(x) \sim \gamma_{\mathrm{V}}^{4} w_{\mathrm{G}}^{2} x^{4}, \\
& \lim _{x \rightarrow \infty} f_{\mathrm{L}}(x) \sim \frac{4 \gamma_{\mathrm{V}}^{4} x^{6}}{w_{\mathrm{G}}^{2}},
\end{aligned}
$$

and

$$
\lim _{x \rightarrow \infty} L_{1}(x) \sim \frac{b_{1 / 2}(a) \exp \left(a^{2}\right) \Phi_{\mathrm{c}}(a) \gamma_{\mathrm{V}}}{\sqrt{\pi} x^{2}} .
$$

It would be worthwhile to point out that, both, $\lim _{x \rightarrow \infty}\left(f_{\mathrm{Q}}(x) Q\right)$ and $\lim _{x \rightarrow \infty}\left(f_{\mathrm{L}}(x) L_{1}(x)\right)$ are of fourth degree on $x$.

Taking into account that $\gamma_{\mathrm{V}}=w_{\mathrm{G}} b_{1 / 2}(a)$, as we see in PI, we obtain

$$
\mu(a, \infty)=\lim _{x \rightarrow \infty} \mu(a, x) \sim \frac{a}{\sqrt{\pi}\left[b_{1 / 2}(a)\right]^{2} \exp \left(a^{2}\right) \Phi_{\mathrm{c}}(a)},
$$

depending on $a$, as expected.

Comparing the $\mu(a)$ values given by the last expression with that obtained from Eq. (11), $\mu(a, \infty)<\mu(a)$ is verified

\section{On the values of the fitting formula
at $x=0$ and $x= \pm \gamma_{\mathrm{V}}$}

Since $G_{1}(x), L_{1}(x)$, and $V_{a}(x)$ take, all of them, by definition, the same value at $x=0$ and $x= \pm \gamma_{\mathrm{V}}$, it is clear that numerator and denominator in Eq. (2) are null at those $x$ values, making it impossible to evaluate $\mu$. 
The denominator of expression (5), $f_{\mathrm{L}}(x) L_{1}(x)+$ $f_{\mathrm{G}}(x) G_{1}(x)$, instead, is null at $x \approx \pm \gamma_{\mathrm{L}}$; please note the symbol $\approx$. For all those $x$ values for which $f_{\mathrm{L}}(x) L_{1}(x)+$ $f_{\mathrm{G}}(x) G_{1}(x)$ is null, $\mu(a, x)$ must not be calculated from Eq. (5).

All the $x$ values for which $\mu(a, x)$ cannot be calculated from Eq. (5), divided by $a \times \gamma_{\mathrm{G}} / \sqrt{\ln 2}$, depend only on $a=w_{L} / w_{\mathrm{G}}$ and are given by the following universal algorithm: we call $\rho(a)$ the function that results after finding the roots of

$$
\frac{f_{L}(x) L_{1}(x)+f_{\mathrm{G}}(x) G_{1}(x}{a \times \gamma_{\mathrm{G}} / \sqrt{\ln 2}} .
$$

$\rho(a)$ can be numerically fitted, with a correlation coefficient of $R^{2}=1$, by the following universal function on $a$ :

$$
\rho(a)=\frac{a_{0}+a_{2} a^{2}+a_{4} a^{4}+a_{6} a^{6}}{1+\beta_{2} a^{2}+\beta_{4} a^{4}+\beta_{6} a^{6}},
$$

where $a_{0}=5.9966, a_{2}=72.0531, a_{4}=-37.0930$, $a_{6}=156.6913, \beta_{2}=102.8163, \beta_{4}=-66.6862$, and $\beta_{6}=156.4950$.

Though $a \times \gamma_{\mathrm{G}} / \sqrt{\ln 2}$ is exactly $w_{\mathrm{L}}$, it is clear that $w_{\mathrm{L}}$ is not an independent parameter entering Eq. (14). $w_{\mathrm{L}}=$ $a \times w_{\mathrm{G}}=a \times \gamma_{\mathrm{G}} / \sqrt{\ln 2}$ enter Eq. (14) only through $a$.

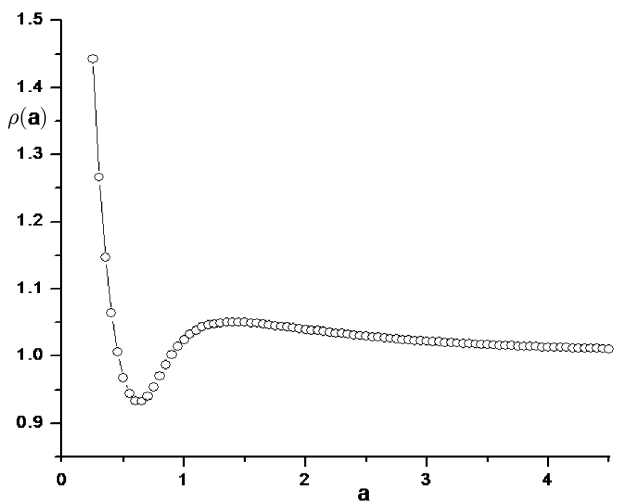

Fig. 1. Roots of $\left[f_{\mathrm{L}}(x) L_{1}(x)+f_{\mathrm{G}}(x) G_{1}(x)\right]$ divided by $w_{\mathrm{L}} \equiv a \gamma_{\mathrm{G}} / \sqrt{\ln 2}$. Points of this plot multiplied by $w_{\mathrm{L}}$ should not be used to calculate $\mu(a, x)$ from Eq. (5) (see text).

$\rho(a)$ is represented in Fig. 1 for $0.25 \leq a \leq 4.5$. For lower or higher $a$ values $V_{a}(x)$ depart very little from a Gaussian and/or a Lorenztian profile, if the noise is $\geq 1 \%$ (easily reached in laboratory experiments).

\section{Indicators of the fit quality}

As it was set in PI, we can test the quality of our fit by normalizing the deviation of $V\left(\mu_{a}, x\right)$ from the exact value

a) to the peak value, $V_{a}(0)$

$$
\Delta_{1}=\frac{V_{a}(x)-V(\mu(a), x)}{V_{a}(0)} \text { or }
$$

or b) to the value at $x, V_{a}(x)$

$$
\Delta_{2}=\frac{V_{a}(x)-V(\mu(a), x)}{V_{a}(x)} .
$$

Criterion (b) is much more sensitive, since, both, the numerator and the denominator of $\Delta_{2}$ are increasingly small numbers.

We are going to see in Sect. 6 that the fit obtained using $V[\mu(a, x), x]$ is better than the one obtained in PI using $V[\mu(a), x]$, specially for high $|x|$ values.

\section{The recipe to construct $\mu(a, x)$}

In order to calculate $\mu(a, x)$, Eqs. (5)-(8) should be used, with $\gamma_{\mathrm{V}}$ obtained from the experimental data. For a selected value of $a$, the needed $w_{\mathrm{G}}$ value is determined by considering the relation between $\gamma_{\mathrm{V}}$ and $w_{\mathrm{G}}$ given by $\gamma_{\mathrm{V}}=w_{\mathrm{G}} b_{1 / 2}(a)$, as it is explained in PI.

The calculation must be done taking into account the algorithm (14), that considers the problem of annulment of both, numerator and denominator cited in Sect. 3 . Then, in first place, $\rho(a)$ must be obtained from Eq. (15) for the selected value of $a$, in order to excluded the values $x \approx \pm \rho(a) \times a \times \gamma_{\mathrm{G}} / \sqrt{\ln 2}\left(\equiv \pm w_{\mathrm{L}} \times \rho(a)\right)$ from the calculations.

Although at this point we have all that is necessary to carry out the calculation of $V_{a}(x) \approx(1-\mu(a, x)) G_{1}(x)+$ $\mu(a, x) L_{1}(x)$, it can be interesting to see the behavior of $\mu(a, x)$, which will be presented in the following section.

\section{Results and discussions}

In Fig. $2 \mu(a, x)$ as a function of $a$, for two different $x$ values, is shown. Note the divergences for $a \approx x / w_{\mathrm{G}}$.

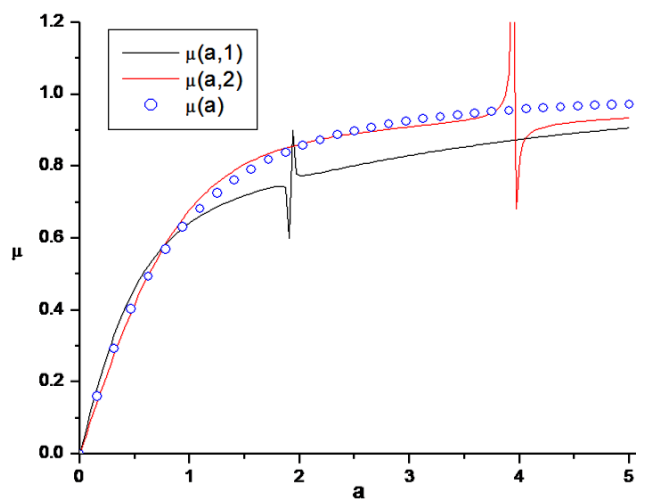

Fig. 2. $\mu(a, x)$ as a function on $a$ for two different $x$ values. $\mu(a, 1)$ and $\mu(a, 2)$ are displayed as functions on $a$ for $w_{\mathrm{G}}=1$. $\mu(a)$, as obtained in PI, is also shown for comparison.

In Fig. $3 \mu(a, x)$ as a function of $x$ is shown, for two different $a$ values. In this case, divergences are observed for $x \approx a w_{\mathrm{G}} \equiv w_{\mathrm{L}}$.

All the curves in Fig. 2 and Fig. 3 have been obtained by adopting $w_{\mathrm{G}}=\gamma_{\mathrm{G}} / \sqrt{\ln 2}=1$ in the calculations, but a similar behavior is observed for all $w_{\mathrm{G}}$ values. In both figures $\mu(a, x)$ is displayed including the divergences, in order to see its real behaviour. In both figures $\mu(a)$, as obtained in PI, is also shown for comparison. 


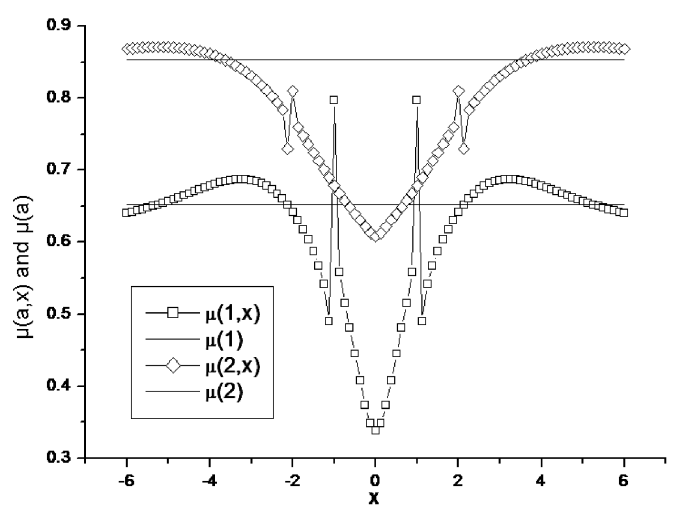

Fig. 3. $\mu(a, x)$ as a function on $x$ for two different $a$ values. $\mu(1, x)$ and $\mu(2, x)$ are displayed as functions on $x$ for $w_{\mathrm{G}}=1 . \mu(1)$ and $\mu(2)$, as obtained in PI, are also shown for comparison.

In Fig. 1 all $x$ values for which $f_{\mathrm{L}}(x) L_{1}(x)+$ $f_{\mathrm{G}}(x) G_{1}(x)$ is null, and, therefore, for which $\mu(a, x)$ cannot be calculated from Eq. (5), are represented (divided by $a \times \gamma_{\mathrm{G}} / \sqrt{\ln 2}$ ) for $0.25 \leq a \leq 4.5$.

In Figs. 4 and 5 we show $\Delta_{1}=\left[V_{a}(x)-V(\mu(a, x), x)\right]$ $/ V_{a}(0)$ and $\Delta_{2}=\left[V_{a}(x)-V(\mu(a, x), x)\right] / V_{a}(x)$ for $\mu$ as a function only on $a$, as we have obtained in PI, and for $\mu$ as a function on $a$ and $x$, as we have obtained in the present paper. It is clear from these figures that an improved fit is obtained when $\mu$ as a function, not only on $a$, but also on $x$, is considered. A good fit is also obtained for high $|x|$ values, which is not obtained by us in PI, nor by other authors in [3] and Liu [6], when $\mu$ as a function only on $a$ is considered.

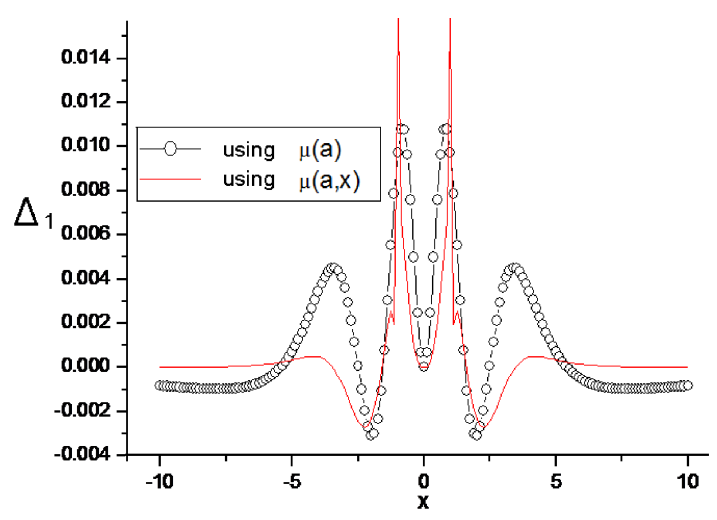

Fig. 4. $\Delta_{1}$ as a function on $|x|$ using $\mu(a)$, as obtained in PI, and $\mu(a, x)$, as obtained at the present paper. The numerical divergences can be observed.

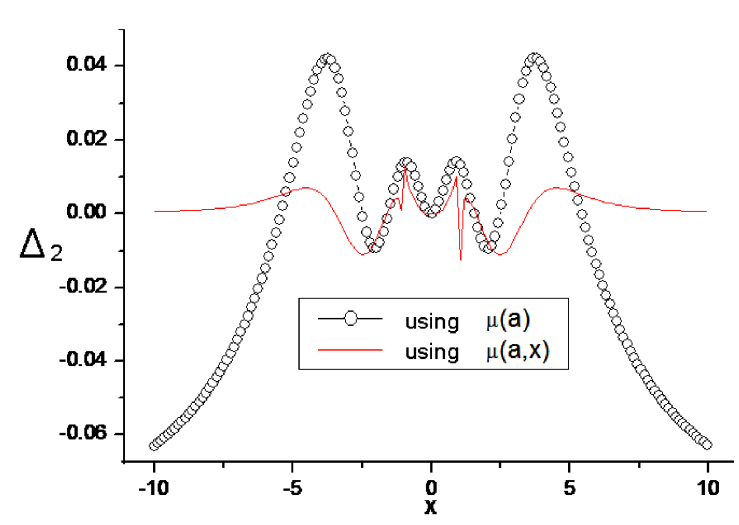

Fig. 5. As in Fig. 4, but for $\Delta_{2}$.

\section{Conclusions}

At the present paper we let $\mu$ to be a function, not only on $a$, but also on $x$. Using one of the differential equations that $V(x)$ satisfies, and neglecting the derivatives $\partial \mu / \partial x$ and $\partial^{2} \mu / \partial x^{2}$ with respect to the derivatives $G_{1}^{\prime}(x), G_{1}^{\prime \prime}(x), L_{1}^{\prime}(x)$, and $L_{1}^{\prime \prime}(x)$, we have been able to obtain: (i) an analytic expression for $\mu(a, x)$, (ii) relative fits, $\Delta_{1}$ and $\Delta_{2}$, better than those we have obtained in PI, (iii) a universal table of values, so that, if we multiply them by $w_{\mathrm{L}} \equiv a \times \gamma_{\mathrm{G}} / \sqrt{\ln 2}$, we obtain the $x$ values for which $\mu(a, x)$ cannot be calculated with the expression we have found.

\section{Acknowledgments}

We wish to thank the Referees for their valuable suggestions that allowed us to significantly improve our article.

\section{References}

[1] H.O. Di Rocco, A. Cruzado, Acta Phys. Pol. A 122, 666 (2012).

[2] H.O. Di Rocco, D.I. Iriarte, J.A. Pomarico, Appl. Spectrosc. 55, 822 (2001).

[3] J.L. Kielkopf, J. Opt. Soc. Am. 63, 987 (1973).

[4] R. Ciurylo, J. Domyslawska, R.S. Trawinski, Acta Phys. Pol. A 83, 425 (1993).

[5] T. Andersen, J. Quant. Spectrosc. Radiat. Transfer 19, 169 (1978).

[6] Y. Liu, J. Lin, G. Huang, Y. Guo, C. Duan, J. Opt. Soc. Am. B 18, 666 (2001). 